\title{
Group Acceptance Sampling Plans using Weighted Binomial on Truncated Life Tests for Inverse Rayleigh and Log - Logistic Distributions
}

\author{
${ }^{1}$ Dr. A. R. Sudamani Ramaswamy, ${ }^{2}$ Priyah Anburajan \\ ${ }^{1}$ Associate Professor and ${ }^{2}$ Research Scholar Department of Mathematics, Avinashilingam University, \\ Coimbatore - 641043, Tamil Nadu, India
}

\begin{abstract}
In this paper, a group acceptance sampling plan using weighted binomial is developed for a truncated life test when the lifetime of an item follows Inverse Rayleigh and Log - Logistic distributions. The minimum number of groups required for a given group size and the acceptance number is determined when the consumer's risk and the test termination time are specified. The operating characteristic values according to various quality levels are obtained. The results are explained with examples.
\end{abstract}

Keywords: Inverse Rayleigh distribution, Log - Logistic distribution, Group acceptance sampling using weighted binomial, consumer's risk, Producer's risk, truncated life test.

\section{Introduction}

Reliability study plays a vital role in the quality control analysis. On the basis of this study, an experimenter can save his time and cost to reach result which is to accept the submitted lot or to reject it. If the genuine products are rejected on the basis of sample information, this error is called type-1. On the other hand, if the genuine products are not accepted by the consumer, this error is type-2 error. If a decision to accept or reject the lot are subjected to the risks associated with the two types of errors, this procedure is termed as reliability test plan or acceptance sampling based on life test. Acceptance sampling is concerned with inspection and decision making regarding lots of product and constitutes one of the oldest techniques in quality assurance. In the 1930s and 1940s acceptance sampling was one of the major components in the field of statistical quality control and was used primarily for incoming or receiving inspection

In most acceptance sampling plans for a truncated life test, determining the sample size from a lot under consideration is the major issue. In the usual sampling plan, it is implicitly assumed that only a single item is put in a tester. However testers accommodating a multiple number of items at a time are used in practise because testing those items simultaneously. Items in a tester can be regarded as a group and the number of items in a group is called as group size. The acceptance sampling plan based on this group of items is called group acceptance sampling plan.

The quality of the product is tested on the basis of few items taken from an infinite lot. The statistical test can be stated as: Let $\mu$ be the true average life and $\mu_{0}$ be the specified average life of a product. Based on the failure data, we want to test the hypothesis $\mathrm{H}_{0}: \mu \geq \mu_{0}$ against $\mathrm{H}_{1}: \mu<\mu_{0}$. A lot is considered as good if $\mu \geq \mu_{0}$ and bad if $\mu<\mu_{0}$. This hypothesis is tested using the acceptance sampling scheme as: In a life test experiment, a sample of size $\mathrm{n}$ selected from a lot of products is put on the test. The experiment is terminated at a pre assigned time $\mathrm{t}_{0}$. when we set acceptance number as $\mathrm{c}, \mathrm{H}_{0}$ is rejected if more than $\mathrm{c}$ failures are recorded before time $t_{0}$ and $\mathrm{H}_{0}$ is accepted if there are $\mathrm{c}$ or fewer failures before $\mathrm{t}_{0}$. Probability of rejection of good lot is called the producer's risk and probability of accepting a bad lot is known as consumer's risk. If the confidence level is $\mathrm{p}^{*}$, then the consumers risk will be $\beta=1-\mathrm{p}^{*}$. A well acceptance sampling plan minimizes both the risks.

Many authors have discussed acceptance sampling based on truncated life tests. Aslam M., and Jun C.H. (2009) have studied a group acceptance sampling plans for truncated life tests based on the inverse Rayleigh and $\log$-logistic distributions. Epstein B. (1954) has discussed truncated life tests in the exponential case. Jun C.H., Balamurali S. and Lee S.-H. (2006) have studied on variables sampling plans for Weibull distributed lifetimes under sudden death testing. Kantam R.R. L., Rosaiah K. and Srinivasa Rao G. (2001) discussed acceptance sampling based on life tests with Log-logistic models. Radhakrishnan R. and Alagirisamy K. (2011) have studied on construction of group acceptance sampling plan using weighted binomial distribution. Rosaiah K. and Kantam R.R.L. (2005) discussed acceptance sampling based on the inverse Rayleigh distribution. Rosaiah K., Kantam R.R.L. and Pratapa Reddy, J. have studied economic reliability test plan with Inverse Rayleigh Variate.

Here we apply group acceptance sampling plans with weighted binomial on the truncated life tests when a lifetime of the product assumed to follow inverse Rayleigh and Log - Logistic distributions. In this type of tests, determining the sample size is equivalent to determining the number of groups. 


\subsection{Inverse Rayleigh Distribution}

\section{Cumulative Distributive Function:}

The cumulative distribution function (cdf) of the Inverse Rayleigh distribution is given by

$$
F(t)=e^{-\frac{\sigma^{2}}{t^{2}}}
$$

where $\sigma$ is a scale parameter.

\subsection{Log - Logistic Distribution}

The cumulative distribution function (cdf) of the Log - Logistic distribution is given by

$$
F(t, \sigma, \theta)=\left[\frac{(t / \sigma)}{1+(t / \sigma)}\right]
$$

where $\sigma$ is a scale parameter.

If some other parameters are involved, then they are assumed to be known, for an example, if shape parameter of a distribution is unknown it is very difficult to design the acceptance sampling plan. In quality control analysis, the scale parameter is often called the quality parameter or characteristics parameter. Therefore it is assumed that the distribution function depends on time only through the ratio of $t / \sigma$.

The failure probability of an item by time $\mathrm{t}_{0}$ is given by

$$
\mathrm{p}=\mathrm{F}\left(\mathrm{t}_{0}: \sigma\right)
$$

The quality of an item is usually represented by its true mean lifetime although some other options such as median life are sometimes used. Let us assume that the true mean $\mu$ can be represented by the scale parameter. In addition, it is convenient to specify the test time as a multiple of the specified life so thata $\mu_{0}$ and the quality of an item as a ratio of the true mean to the specified life $\left(\mu / \mu_{0}\right)$.

Then we can rewrite (3) as a function of 'a' (termination time) and the ratio $\mu / \mu_{0}$.

$$
\mathrm{p}=\mathrm{F}\left(\mathrm{a} \mu_{0}: \mu / \mu_{0}\right)
$$

Here when the underlying distribution is the Inverse Rayleigh distribution

$$
p=\exp \left(-\frac{1}{a^{2} \pi}\left(\frac{\mu}{\mu_{0}}\right)^{2}\right)
$$

When the underlying distribution is the $\log -$ Logistic distribution

$$
p=\frac{(1.5708 a)^{2}}{\left(\mu / \mu_{0}\right)^{2}+(1.5708 a)^{2}}
$$

\subsection{Procedure:}

\section{Design of the proposed sampling plan:}

1) Select the number of groups $g$ and allocate predefined $r$ items to each group so that the sample size for a lot will be $n=g r$.

2) Select the acceptance number $c$ for a group and specify the experiment time $t_{0}$.

3) Perform the experiment for the $g$ groups simultaneously and record the number of failures for each group.

4) Accept the lot if at most $c$ failures occurs in each of all groups by the experiment time.

5) Terminate the experiment as soon as more than $c$ failures occur in any group and reject the lot.

We are interested in determining the number of group g, whereas the various values of acceptance number $\mathrm{c}$ and the termination time $\mathrm{t}_{0}$ are assumed to be specified. Since it is convenient to set the termination time as a multiple of the specified life $\mu_{0}$, we will consider $t_{0}=a \mu_{0}$ for a specified constant a (time multiplier). The lot acceptance probability will be

$$
L(p)=\left(\sum_{i=1}^{c}\left(\begin{array}{c}
r-1 \\
i-1
\end{array}\right) p^{i-1}(1-p)^{r-1}\right)^{g}
$$

where $p$ is the probability that an item in a group fails before the termination time $t_{0}=a \mu_{0}$.

The minimum number of groups required can be determined by considering the consumer's risk when the true median life equals the specified median life $\left(\mu=\mu_{0}\right)$ (worst case) by means of the following inequality:

$$
\boldsymbol{L}\left(\boldsymbol{p}_{\mathrm{o}}\right) \leq \boldsymbol{\beta}
$$


where $p_{0}$ is the failure probability at $\mu=\mu_{0}$. Here minimum group size (g) is obtained using (5) and (6) in (8) at worst case.

IV. Operating Characteristic functions:

The probability of acceptance can be regarded as a function of the deviation of the specified value $\mu_{0}$ of the median from its true value $\mu$. This function is called Operating Characteristic (OC) function of the sampling plan. Once the minimum sample size is obtained, one may be interested to find the probability of acceptance of a lot when the quality (or reliability) of the product is sufficiently good. As mentioned earlier, the product is considered to be good if $\mu \geq \mu_{0}$. Te probabilities of acceptance are displayed in Table 3 and 4 for various values of the median ratios $\mu / \mu_{0}$, producer's risks $\beta$ and time multiplier $a$.

\begin{tabular}{lll} 
& & \multicolumn{1}{c}{ V. } \\
$\mathrm{g}$ & - & Number of groups \\
$\mathrm{r}$ & - & Number of items in a group \\
$\mathrm{n}$ & - & Sample size \\
$\mathrm{c}$ & - & Acceptance number \\
$\mathrm{t}_{0}$ & - & Termination time \\
$\mathrm{a}$ & - & Test termination time multiplier \\
$\sigma$ & - & Scale parameter \\
$\alpha$ & - & Producer's risk \\
$\beta$ & - & Consumer's risk \\
$p$ & - & Failure probability \\
$\mathrm{L}(p)$ & - & Probability of acceptance \\
$\mu$ & - & Mean life \\
$\mu_{0}$ & - & Specified life
\end{tabular}

\section{Description of tables and examples:}

Based on various test values of consumer's risk and the test termination time multiplier, the number of groups of GASP is found using (5), (6), (7) and (8). Suppose that we want to develop an economic reliability group sampling plan to test if the median is greater than 1,000 hours based on a testing time of 700 hours and using testers equipped with 6 items each. It is assumed that $\mathrm{c}=2$ and $\beta=0.1$. This gives the termination multiplier $a=0.7$. If the life time follows inverse Rayleigh distribution, from Table 1 the design parameters can be written as $(g, c)=(3,2)$. We will implement the above sampling plan as, draw the first sample of size $n=18$ items and put to 6 testers, if not more than 2 failures occur during 700 hours in any one of the groups, we accept the lot, otherwise reject it. If suppose the life time follows Log - Logistic distribution, from Table 2 the design parameters can be written as $(\mathrm{g}, \mathrm{c})=(3,2)$ which is similar as the above. For this proposed sampling plan if $\mathrm{r}=$ $4, \beta=0.25$ and the life time follows inverse Rayleigh distribution, the probability of acceptance is 0.9959 when the true mean is $2000 \mathrm{hrs}$ from Table 3 and if the life time follows Log - Logistic distribution the probability of acceptance is 0.9043 when the true mean is $2000 \mathrm{hrs}$ from Table 4 . When the probability of acceptance is compared inverse Rayleigh distribution has more probability of acceptance than the Log - Logistic distribution. Thus inverse Rayleigh distribution is comparatively good for the group acceptance sampling plan with weighted binomial. When comparing the probability of acceptance of Aslam and Jun[1], for the same, it is 0.9938 for inverse Rayleigh distribution and 0.8813 for Log - Logistic distribution for group sampling plans. It is observed that the lot acceptance probability increases as the mean ratio increases and the number of groups tends to increase as the test duration decreases and is shown in Figure 1.

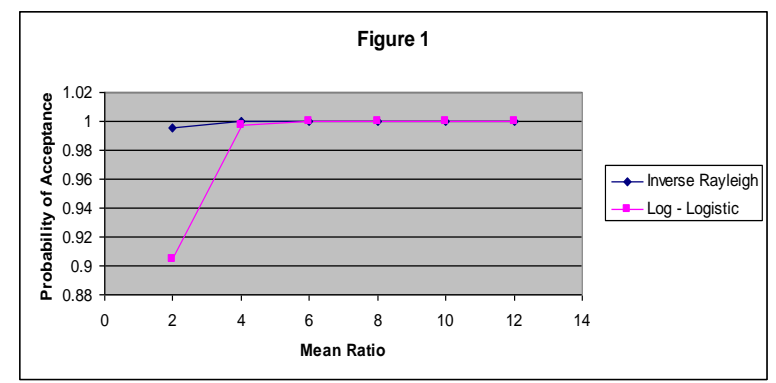

Figure 1. OC curve for Probability of acceptance against mean ratio 


\section{Conclusion}

In this paper, a group acceptance sampling plan from a truncated life test is proposed in the case of a inverse Rayleigh and Log - Logistic distributions. The number of groups and the acceptance number are determined when the consumer's risk $(\beta)$ and the other plan parameters are specified. It is observed that the minimum number of groups required decreases as the test termination time multiplier increases. Moreover, the operating characteristic function increases disproportionately when the quality improves. This group acceptance sampling plan with weighted binomial can be used when a multiple number of items are tested simultaneously. Clearly, such a tester would be beneficial in terms of test time and test cost.

Table 1: Minimum number of groups (g) for the proposed plan in case of Inverse Rayleigh distribution

\begin{tabular}{|c|c|c|c|c|c|c|c|c|}
\hline $\bar{\beta}$ & $\mathbf{r}$ & c & $\begin{array}{l}\text { a } \\
\text { o. } 7\end{array}$ & 0.8 & 1.0 & 1.2 & 1.5 & 2.0 \\
\hline 0.25 & 2 & 0 & 2 & 2 & 2 & 1 & $\overline{1}$ & $\overline{1}$ \\
\hline 0.25 & 3 & 1 & 5 & 4 & 2 & 2 & 1 & 1 \\
\hline 0.25 & 4 & 2 & 10 & 6 & 3 & 2 & 2 & 1 \\
\hline 0.25 & 5 & 3 & 18 & 10 & 5 & 3 & 2 & 2 \\
\hline 0.25 & 6 & 4 & 35 & 16 & 7 & 4 & 3 & 2 \\
\hline 0.25 & 7 & 5 & 69 & 27 & 9 & 5 & 3 & 2 \\
\hline 0.10 & 4 & o & 1 & 1 & 1 & 1 & 1 & 1 \\
\hline 0.10 & 5 & 1 & 2 & 2 & 1 & 1 & 1 & 1 \\
\hline 0.10 & 6 & 2 & 3 & 2 & 2 & 1 & 1 & 1 \\
\hline 0.10 & 7 & 3 & 5 & 3 & 2 & 1 & 1 & 1 \\
\hline 0.10 & 8 & 4 & 8 & 4 & 2 & 2 & 1 & 1 \\
\hline 0.10 & 9 & 5 & 12 & 6 & 3 & 2 & 1 & 1 \\
\hline 0.05 & 5 & o & 1 & 1 & 1 & 1 & 1 & 1 \\
\hline 0.05 & 6 & 1 & 2 & 2 & 1 & 1 & 1 & 1 \\
\hline 0.05 & 7 & 2 & 3 & 2 & 2 & 1 & 1 & 1 \\
\hline 0.05 & 8 & 3 & 4 & 3 & 2 & 1 & 1 & 1 \\
\hline 0.05 & 9 & 4 & 6 & 4 & 2 & 2 & 1 & 1 \\
\hline 0.05 & 10 & 5 & 9 & 5 & 2 & 2 & 1 & 1 \\
\hline 0.01 & 7 & o & 1 & 1 & 1 & 1 & 1 & 1 \\
\hline 0.01 & 8 & 1 & 2 & 2 & 1 & 1 & 1 & 1 \\
\hline 0.01 & 9 & 2 & 3 & 2 & 1 & 1 & 1 & 1 \\
\hline 0.01 & 10 & 3 & 3 & 2 & 2 & 1 & 1 & 1 \\
\hline 0.01 & 11 & 4 & 5 & 3 & 2 & 1 & 1 & 1 \\
\hline 0.01 & 12 & 5 & 6 & 4 & 2 & 2 & 1 & 1 \\
\hline
\end{tabular}

Table 2: Minimum number of groups ( $\mathrm{g}$ ) for the proposed plan in case of Log - Logistic distribution

\begin{tabular}{|c|c|c|c|c|c|c|c|c|}
\hline F & $\mathbf{r}$ & $c$ & a. 7 & 0.8 & 1.0 & 1.2 & 1.5 & 2.0 \\
\hline 0.25 & 2 & 0 & 2 & 2 & 2 & 1 & 1 & 1 \\
\hline 0.25 & 3 & 1 & 4 & 3 & 2 & 2 & 2 & 1 \\
\hline 0.25 & 4 & 2 & 8 & 6 & 4 & 3 & 2 & 2 \\
\hline 0.25 & 5 & 3 & 15 & 10 & 5 & 3 & 2 & 2 \\
\hline 0.25 & 6 & 4 & 28 & 16 & 9 & 5 & 3 & 2 \\
\hline 0.25 & 7 & 5 & 51 & 26 & 10 & 6 & 3 & 2 \\
\hline 0.10 & 4 & $\mathbf{0}$ & 1 & 1 & 1 & 1 & 1 & 1 \\
\hline 0.10 & 5 & 1 & 2 & 2 & 1 & 1 & 1 & 1 \\
\hline 0.10 & 6 & 2 & 3 & 2 & 2 & 1 & 1 & 1 \\
\hline 0.10 & 7 & 3 & 5 & 3 & 2 & 2 & 1. & 1 \\
\hline 0.10 & 8 & 4 & 7 & 4 & 3 & 2 & 1 & 1 \\
\hline 0.10 & 9 & 5 & 10 & 6 & 3 & 2 & 2 & 1 \\
\hline 0.05 & 5 & 0 & 1 & 1 & 1 & 1 & 1 & 1 \\
\hline 0.05 & 6 & 1 & 2 & 2 & 1 & 1 & 1 & 1 \\
\hline 0.05 & 7 & 2 & 3 & 2 & 2 & 1 & 1 & 1 \\
\hline 0.05 & 8 & 3 & 4 & 3 & 2 & 1 & 1 & 1 \\
\hline 0.05 & 9 & 4 & 5 & 4 & 2 & 2 & 1 & 1 \\
\hline 0.05 & 10 & 5 & 7 & 5 & 3 & 2 & 1 & 1 \\
\hline 0.01 & 7 & $\mathbf{0}$ & 1 & 1 & 1 & 1 & 1 & 1 \\
\hline 0.01 & 8 & 1 & 2 & 2 & 1 & 1 & 1 & 1 \\
\hline 0.01 & 9 & 2 & 2 & 2 & 1 & 1 & 1 & 1 \\
\hline 0.01 & 10 & 3 & 3 & 2 & 2 & 1 & 1 & 1 \\
\hline 0.01 & 11 & 4 & 4 & 3 & 2 & 2 & 1. & 1. \\
\hline 0.01 & 12 & 5 & 5 & 4 & 2 & 2 & 1 & 1 \\
\hline
\end{tabular}


Group Acceptance Sampling Plans using Weighted Binomial on Truncated Life Tests for Inverse Table 3: Operating characteristic values of the group sampling plan with $\mathrm{c}=2$ for Inverse Rayleigh distribution

\begin{tabular}{|l|lllllllll|}
\hline $\boldsymbol{\beta}$ & $\mathbf{r}$ & $\mathbf{a}$ & $\mathbf{g}$ & $\mathbf{\mu} / \boldsymbol{\mu}_{0}$ & $\mathbf{4}$ & $\mathbf{6}$ & $\mathbf{8}$ & $\mathbf{1 0}$ & $\mathbf{1 2}$ \\
\hline $\mathbf{0 . 2 5}$ & $\mathbf{4}$ & $\mathbf{0 . 7}$ & $\mathbf{1 0}$ & 0.995878 & 1.000000 & 1.000000 & 1.000000 & 1.000000 & 1.000000 \\
$\mathbf{0 . 2 5}$ & $\mathbf{4}$ & $\mathbf{0 . 8}$ & $\mathbf{6}$ & 0.984710 & 1.000000 & 1.000000 & 1.000000 & 1.000000 & 1.000000 \\
$\mathbf{0 . 2 5}$ & $\mathbf{4}$ & $\mathbf{1 . 0}$ & $\mathbf{3}$ & 0.935534 & 0.999999 & 1.000000 & 1.000000 & 1.000000 & 1.000000 \\
$\mathbf{0 . 2 5}$ & $\mathbf{4}$ & $\mathbf{1 . 2}$ & $\mathbf{2}$ & 0.863888 & 0.999950 & 1.000000 & 1.000000 & 1.000000 & 1.000000 \\
$\mathbf{0 . 2 5}$ & $\mathbf{4}$ & $\mathbf{1 . 5}$ & $\mathbf{2}$ & 0.667100 & 0.997747 & 1.000000 & 1.000000 & 1.000000 & 1.000000 \\
$\mathbf{0 . 2 5}$ & $\mathbf{4}$ & $\mathbf{2 . 0}$ & $\mathbf{1}$ & 0.615013 & 0.978032 & 0.999814 & 1.000000 & 1.000000 & 1.000000 \\
\hline $\mathbf{0 . 1 0}$ & $\mathbf{6}$ & $\mathbf{0 . 7}$ & $\mathbf{3}$ & 0.988995 & 1.000000 & 1.000000 & 1.000000 & 1.000000 & 1.000000 \\
$\mathbf{0 . 1 0}$ & $\mathbf{6}$ & $\mathbf{0 . 8}$ & $\mathbf{2}$ & 0.959088 & 1.000000 & 1.000000 & 1.000000 & 1.000000 & 1.000000 \\
$\mathbf{0 . 1 0}$ & $\mathbf{6}$ & $\mathbf{1 . 0}$ & $\mathbf{2}$ & 0.743511 & 0.999995 & 1.000000 & 1.000000 & 1.000000 & 1.000000 \\
$\mathbf{0 . 1 0}$ & $\mathbf{6}$ & $\mathbf{1 . 2}$ & $\mathbf{1}$ & 0.659521 & 0.999763 & 1.000000 & 1.000000 & 1.000000 & 1.000000 \\
$\mathbf{0 . 1 0}$ & $\mathbf{6}$ & $\mathbf{1 . 5}$ & $\mathbf{1}$ & 0.374087 & 0.990413 & 0.999998 & 1.000000 & 1.000000 & 1.000000 \\
$\mathbf{0 . 1 0}$ & $\mathbf{6}$ & $\mathbf{2 . 0}$ & $\mathbf{1}$ & 0.128689 & 0.862271 & 0.998298 & 0.999998 & 1.000000 & 1.000000 \\
\hline $\mathbf{0 . 0 5}$ & $\mathbf{7}$ & $\mathbf{0 . 7}$ & $\mathbf{3}$ & 0.979278 & 1.000000 & 1.000000 & 1.000000 & 1.000000 & 1.000000 \\
$\mathbf{0 . 0 5}$ & $\mathbf{7}$ & $\mathbf{0 . 8}$ & $\mathbf{2}$ & 0.927060 & 1.000000 & 1.000000 & 1.000000 & 1.000000 & 1.000000 \\
$\mathbf{0 . 0 5}$ & $\mathbf{7}$ & $\mathbf{1 . 0}$ & $\mathbf{2}$ & 0.608867 & 0.999991 & 1.000000 & 1.000000 & 1.000000 & 1.000000 \\
$\mathbf{0 . 0 5}$ & $\mathbf{7}$ & $\mathbf{1 . 2}$ & $\mathbf{1}$ & 0.516978 & 0.999536 & 1.000000 & 1.000000 & 1.000000 & 1.000000 \\
$\mathbf{0 . 0 5}$ & $\mathbf{7}$ & $\mathbf{1 . 5}$ & $\mathbf{1}$ & 0.226344 & 0.982306 & 0.999995 & 1.000000 & 1.000000 & 1.000000 \\
$\mathbf{0 . 0 5}$ & $\mathbf{7}$ & $\mathbf{2 . 0}$ & $\mathbf{1}$ & 0.050762 & 0.780300 & 0.996740 & 0.999995 & 1.000000 & 1.000000 \\
\hline $\mathbf{0 . 0 1}$ & $\mathbf{9}$ & $\mathbf{0 . 7}$ & $\mathbf{3}$ & 0.948731 & 1.000000 & 1.000000 & 1.000000 & 1.000000 & 1.000000 \\
$\mathbf{0 . 0 1}$ & $\mathbf{9}$ & $\mathbf{0 . 8}$ & $\mathbf{2}$ & 0.838487 & 1.000000 & 1.000000 & 1.000000 & 1.000000 & 1.000000 \\
$\mathbf{0 . 0 1}$ & $\mathbf{9}$ & $\mathbf{1 . 0}$ & $\mathbf{1}$ & 0.602558 & 0.999987 & 1.000000 & 1.000000 & 1.000000 & 1.000000 \\
$\mathbf{0 . 0 1}$ & $\mathbf{9}$ & $\mathbf{1 . 2}$ & $\mathbf{1}$ & 0.288435 & 0.998758 & 1.000000 & 1.000000 & 1.000000 & 1.000000 \\
$\mathbf{0 . 0 1}$ & $\mathbf{9}$ & $\mathbf{1 . 5}$ & $\mathbf{1}$ & 0.072699 & 0.957745 & 0.999987 & 1.000000 & 1.000000 & 1.000000 \\
$\mathbf{0 . 0 1}$ & $\mathbf{9}$ & $\mathbf{2 . 0}$ & $\mathbf{1}$ & 0.006751 & 0.602558 & 0.991628 & 0.999987 & 1.000000 & 1.000000 \\
\hline
\end{tabular}

Table 4: Operating characteristic values of the group sampling plan with $c=2$ for $\log -$ Logistic distribution

\begin{tabular}{|l|lllllllll|}
\hline $\boldsymbol{\beta}$ & $\mathbf{r}$ & $\mathbf{a}$ & $\mathbf{g}$ & $\mathbf{\mu} / \boldsymbol{\mu}_{\mathbf{0}}$ & $\mathbf{4}$ & $\mathbf{6}$ & $\mathbf{8}$ & $\mathbf{1 0}$ & $\mathbf{1 2}$ \\
\hline $\mathbf{0 . 2 5}$ & $\mathbf{4}$ & $\mathbf{0 . 7}$ & $\mathbf{8}$ & 0.904239 & 0.997229 & 0.999726 & 0.999949 & 0.999986 & 0.999995 \\
$\mathbf{0 . 2 5}$ & $\mathbf{4}$ & $\mathbf{0 . 8}$ & $\mathbf{6}$ & 0.871429 & 0.995659 & 0.999555 & 0.999916 & 0.999977 & 0.999992 \\
$\mathbf{0 . 2 5}$ & $\mathbf{4}$ & $\mathbf{1 . 0}$ & $\mathbf{4}$ & 0.795703 & 0.990494 & 0.998945 & 0.999795 & 0.999944 & 0.999981 \\
$\mathbf{0 . 2 5}$ & $\mathbf{4}$ & $\mathbf{1 . 2}$ & $\mathbf{3}$ & 0.719088 & 0.982107 & 0.997827 & 0.999564 & 0.999879 & 0.999958 \\
$\mathbf{0 . 2 5}$ & $\mathbf{4}$ & $\mathbf{1 . 5}$ & $\mathbf{2}$ & 0.645851 & 0.966105 & 0.995235 & 0.998983 & 0.999709 & 0.999898 \\
$\mathbf{0 . 2 5}$ & $\mathbf{4}$ & $\mathbf{2 . 0}$ & $\mathbf{2}$ & 0.409169 & 0.892022 & 0.980176 & 0.995235 & 0.998551 & 0.999472 \\
\hline $\mathbf{0 . 1 0}$ & $\mathbf{6}$ & $\mathbf{0 . 7}$ & $\mathbf{3}$ & 0.764687 & 0.990691 & 0.999021 & 0.999814 & 0.999950 & 0.999983 \\
$\mathbf{0 . 1 0}$ & $\mathbf{6}$ & $\mathbf{0 . 8}$ & $\mathbf{2}$ & 0.737221 & 0.987425 & 0.998608 & 0.999731 & 0.999927 & 0.999975 \\
$\mathbf{0 . 1 0}$ & $\mathbf{6}$ & $\mathbf{1 . 0}$ & $\mathbf{2}$ & 0.509772 & 0.961721 & 0.995222 & 0.999033 & 0.999731 & 0.999907 \\
$\mathbf{0 . 1 0}$ & $\mathbf{6}$ & $\mathbf{1 . 2}$ & $\mathbf{1}$ & 0.555344 & 0.955164 & 0.993693 & 0.998657 & 0.999617 & 0.999865 \\
$\mathbf{0 . 1 0}$ & $\mathbf{6}$ & $\mathbf{1 . 5}$ & $\mathbf{1}$ & 0.350361 & 0.888309 & 0.980674 & 0.995504 & 0.998657 & 0.999516 \\
$\mathbf{0 . 1 0}$ & $\mathbf{6}$ & $\mathbf{2 . 0}$ & $\mathbf{1}$ & 0.148076 & 0.713983 & 0.929768 & 0.980674 & 0.993693 & 0.997608 \\
\hline $\mathbf{0 . 0 5}$ & $\mathbf{7}$ & $\mathbf{0 . 7}$ & $\mathbf{3}$ & 0.631265 & 0.982406 & 0.998089 & 0.999633 & 0.999900 & 0.999966 \\
$\mathbf{0 . 0 5}$ & $\mathbf{7}$ & $\mathbf{0 . 8}$ & $\mathbf{2}$ & 0.600699 & 0.976593 & 0.997305 & 0.999471 & 0.999855 & 0.999950 \\
$\mathbf{0 . 0 5}$ & $\mathbf{7}$ & $\mathbf{1 . 0}$ & $\mathbf{2}$ & 0.339429 & 0.931538 & 0.990911 & 0.998121 & 0.999471 & 0.999816 \\
$\mathbf{0 . 0 5}$ & $\mathbf{7}$ & $\mathbf{1 . 2}$ & $\mathbf{1}$ & 0.400729 & 0.922288 & 0.988227 & 0.997420 & 0.999253 & 0.999736 \\
$\mathbf{0 . 0 5}$ & $\mathbf{7}$ & $\mathbf{1 . 5}$ & $\mathbf{1}$ & 0.206157 & 0.818365 & 0.965162 & 0.991541 & 0.997420 & 0.999060 \\
$\mathbf{0 . 0 5}$ & $\mathbf{7}$ & $\mathbf{2 . 0}$ & $\mathbf{1}$ & 0.061640 & 0.582605 & 0.881611 & 0.965162 & 0.988227 & 0.995445 \\
\hline $\mathbf{0 . 0 1}$ & $\mathbf{9}$ & $\mathbf{0 . 7}$ & $\mathbf{2}$ & 0.522022 & 0.970529 & 0.996605 & 0.999334 & 0.999995 & 0.999937 \\
$\mathbf{0 . 0 1}$ & $\mathbf{9}$ & $\mathbf{0 . 8}$ & $\mathbf{2}$ & 0.353987 & 0.943340 & 0.992926 & 0.998573 & 0.999992 & 0.999863 \\
$\mathbf{0 . 0 1}$ & $\mathbf{9}$ & $\mathbf{1 . 0}$ & $\mathbf{1}$ & 0.355187 & 0.920553 & 0.988430 & 0.997511 & 0.999981 & 0.999749 \\
$\mathbf{0 . 0 1}$ & $\mathbf{9}$ & $\mathbf{1 . 2}$ & $\mathbf{1}$ & 0.186844 & 0.835706 & 0.971257 & 0.993329 & 0.999958 & 0.999286 \\
$\mathbf{0 . 0 1}$ & $\mathbf{9}$ & $\mathbf{1 . 5}$ & $\mathbf{1}$ & 0.062467 & 0.659521 & 0.920553 & 0.989026 & 0.999898 & 0.997511 \\
$\mathbf{0 . 0 1}$ & $\mathbf{9}$ & $\mathbf{2 . 0}$ & $\mathbf{1}$ & 0.009151 & 0.355187 & 0.762626 & 0.920553 & 0.999472 & 0.988430 \\
\hline
\end{tabular}




\section{References}

[1] Aslam, M., and Jun, C.-H. (2009): A group acceptance sampling plans for truncated life tests based on the inverse Rayleigh and loglogistic distributions. Pakistan Journal of Statistics 25, 1-13.

[2] Epstein, B. (1954): Truncated life tests in the exponential case. Annals of Mathematical Statistics 25, 555-564.

[3] Jun, C.H., Balamurali, S. and Lee, S.-H. (2006): Variables sampling plans for Weibull distributed lifetimes under sudden death testing. IEEE Transactions on Reliability 55, 53-58.

[4] Kantam, R.R. L., Rosaiah, K. and Srinivasa Rao, G. (2001): Acceptance sampling based on life tests: Log-logistic models. Journal of Applied Statistics 28, 121-128.

[5] Radhakrishnan R. and Alagirisamy K. (2011): Construction of group acceptance sampling plan using weighted binomial distribution. International Journal of Recent Scientific Research 2(7), 229-231.

[6] Rosaiah, K. and Kantam, R.R.L. (2005): Acceptance sampling based on the inverse Rayleigh distribution. EQC 20, $277-286$.

[7] Rosaiah, K., Kantam, R.R.L. and Pratapa Reddy, J. (2007): Economic reliability test plan with Inverse Rayleigh Variate. Pakistan Journal of Statistics 24, 57-65. 